\title{
Role of minimally invasive surgery versus open approach in patients with early-stage uterine carcinosarcomas: a retrospective multicentric study
}

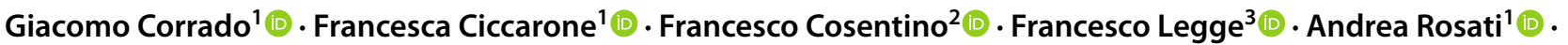

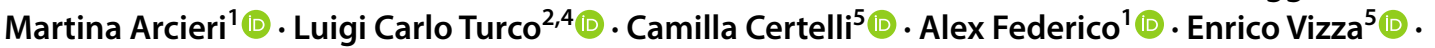 \\ Francesco Fanfani ${ }^{1,6}\left(\mathbb{0} \cdot\right.$ Giovanni Scambia $^{1,6}(\mathbb{0}) \cdot$ Gabriella Ferrandina ${ }^{1,6}(\mathbb{1})$
}

Received: 18 July 2020 / Accepted: 19 August 2020 / Published online: 3 September 2020

(c) The Author(s) 2020

\begin{abstract}
Objective The aim of this retrospective study was to compare surgical and survival outcome in only patients with early-stage UCSs managed by laparotomic surgery (LPT) versus minimally invasive surgery (MIS).

Methods Data were retrospectively collected in four Italian different institutions. Inclusion criteria were UCS diagnosis confirmed by the definitive histological examination, and stage I or II according to the FIGO staging system.

Results Between August 2000 and March 2019, the data relative to 170 patients bearing UCSs were collected: of these, 95 were defined as early-stage disease (stage I-II) based on the histological report at the primary surgery, and thus were included in this study. Forty-four patients were managed by LPT, and 51 patients were managed by MIS. The operative time was lower in the MIS group versus the LPT group ( $p$ value 0.021 ); the median estimated blood loss was less in the MIS group compared to the median of LPT group ( $p$ value $<0.0001$ ). The length of hospital stay days was shorter in the MIS patients ( $p$ value $<0.0001)$. Overall, there were eight $(8.4 \%)$ post-operative complications; of these, seven were recorded in the LPT group versus one in the MIS group ( $p$ value 0.023 ). There was no difference in the disease-free survival (DFS) and overall survival (OS) between the two groups.

Conclusion There was no difference of oncologic outcome between the two approaches, in face of a more favourable perioperative and post-operative profile in the MIS group.
\end{abstract}

Keywords Uterine carcinosarcoma $\cdot$ Minimally invasive surgery $\cdot$ Open surgery

Giacomo Corrado

giacomo.corrado@alice.it

1 Department of Woman, Child Health and Public Health, Gynecologic Oncology Unit, Fondazione Policlinico

Universitario A. Gemelli, IRCCS, Rome, Italy

2 Department of Gynecologic Oncology, Gemelli Molise Spa, Università Cattolica del Sacro Cuore, Campobasso, Italy

3 Gynecologic Oncology Unit, "F. Miulli” General Regional Hospital, Acquaviva Delle Fonti, Bari, Italy

4 Department Gynecology and Breast Care Unit, Mater Olbia Spa, Olbia, Italy

5 Department of Experimental Clinical Oncology, Gynecologic Oncology Unit, "Regina Elena" National Cancer Institute, IRCCS, Rome, Italy

6 Istituto di Ostetricia e Ginecologia, Fondazione Policlinico Universitario A. Gemelli, IRCCS, Università Cattolica del Sacro Cuore, Rome, Italy

\section{Introduction}

Uterine carcinosarcomas (UCSs) are rare and aggressive malignancies characterized by the concomitant presence of carcinomatous and sarcomatous components (Akahira et al. 2006; Cantrell et al. 2015). UCSs account for less than 5\% of all uterine tumors, however, a recent study conducted in the USA has reported that the rate of this disease has significantly increased over time, possibly due to the increase of number of older patients, obesity, increased use of tamoxifen, etc. (Pothuri et al. 2006; Onstad et al. 2016; Matsuo et al. 2018). Even though UCSs are considered as metaplastic endometrial carcinomas (EC), their aggressiveness is much higher compared to the classical EC; indeed, the proportion of stage I-II is lower in UCSs compared to EC, and, even in early stage, UCSs patients experience worse 
prognosis with a 5-years survival of $60 \%$ (Gonzalez Bosquet et al. 2010).

In addition, high grade, older age, and lymphovascular space invasion are more frequently documented in UCSs than in other EC types (Cantrell et al. 2015; Abdulfatah et al. 2019). In this context, it has also to be acknowledged that UCSs display a mutational profile endowed with high copy number, and unfavourable clinical outcome (The Cancer Genome Atlas Research Network and Levine 2013; Leskela et al. 2019; Carlson and McCluggage 2019).

In early-stage disease, surgery represents the milestone of treatment, and includes total hysterectomy, bilateral salpingo-oophorectomy, pelvic and aortic lymphadenectomy, and peritoneal biopsies (Baekelandt and Castiglione 2009; Denschlag and Ulrich 2018) www.ncenguidelines.gov (2020); even though omentectomy or omental al sampling are not formally recommended, these procedures often carried out probably because microscopic involvement has been shown to account for $35 \%$ of omental disease, and thus could be missed (Ross et al. 2018).

The most frequent surgical approach adopted in early UCSs is laparotomy, and few retrospective data are available relative to the minimally invasive surgery in UCSs (Wallwiener et al. 2016; Tan et al. 2017; Dellinger et al. 2017); within the GOG LAP2 trial (Walker et al. 2009), which has investigated the clinical outcome of early-stage EC patients triaged to laparoscopic versus laparotomic surgery, the sub-group analysis of "high risk" histotypes (i.e. clear cells, serous, and UCSs) reported similar rates of recurrence and survival between the two approaches (Fader et al. 2016).

The aim of this retrospective study was to compare surgical and survival outcome in only patients with early-stage UCSs managed by laparotomic surgery (LPT) versus minimally invasive surgery (MIS).

\section{Patients and methods}

Data were retrospectively collected in four different institutions: "Fondazione Policlinico Universitario A. Gemelli" Rome, "Regina Elena National Cancer Institute" Rome, "Gemelli Molise spa, Università Cattolica del Sacro Cuore" Campobasso, and the "Miulli hospital", Acquaviva delle Fonti in Bari. This study was approved by the internal review board at each participating institution. All patients had already provided a written informed consent for their data to be collected and analysed for scientific purpose, according to our institutional policy.

Inclusion criteria were UCS diagnosis confirmed by the definitive histological examination, and stage I or II according to the FIGO staging system (Creasman 2009).

Patients with anesthesiological contraindications for the MIS approach (i.e. patients who could not sustain a steep
Trendeleburg position) were triaged to laparotomic surgery. Previous abdominal surgery was not considered an exclusion criterion.

All patients were evaluated before surgery by means of medical history, physical examination, vaginal-pelvic examination, chest X-ray or chest CT, pelvic ultrasound scans and complete abdomen and pelvis CT scan or MRI; PET/ CT scans if any suspicious images in previous examinations.

Details relative to the type of hysterectomy according to the Querleu-Morrow classification (Querleu and Morrow 2008), and lymph node assessment (i.e. systematic lymphadenectomy or lymph node sampling or sentinel lymph node technique (SLN) were collected in both groups.

Intraoperative and postoperative complications were defined according to Common Terminology Criteria for Adverse Events (CTCAE) version 4.3 (U.S. Department of Health and Human Services 2009).

Adjuvant therapy was tailored to the pathologic findings at the primary surgery after multidisciplinary tumor board (gynecologic oncology, pathology, radiation oncology, medical oncology) discussion. Adjuvant chemotherapy including always platinum/paclitaxel or just platinum in unfit patients. The vast majority of adjuvant radiotherapy was represented by external beam pelvic irradiation (45-50 Gy) plus brachytherapy in case of cervical involvement. Treatment was based on the National Comprehensive Cancer Network (NCCN) guidelines (www.nccn.org.professionals.physician_gls) as well as ESGO, and ESTRO guidelines (Baekelandt and Castiglione 2009; Creasman 2009; Plataniotis and Castiglione 2010; Colombo et al. 2016).

The data relative to the time interval from surgery to the start of adjuvant therapy were calculated, if available. Follow-up data were recorded through phone calls, if not available from medical records.

Follow-up schedule include clinical and gynecological examination, CA125 level and ultrasound pelvic scan every 3 months in the first 2 years and every 6 months thereafter; abdomen and thorax CT scan after the end of adjuvant treatment and every 6 months in the first 2 years and every 12 months thereafter. In case of undefined lesions to ultrasound or CT scan, it is scheduled a PET/CT.

\section{Statistical analysis}

Descriptive analysis of data was carried out by Fisher's exact test for proportion for categorical data or Wilcoxon rank sum non-parametric test for continuous variables. Disease-free survival (DFS) would be calculated from the date of surgery up to disease progression or the date last seen; overall survival (OS) would be calculated from the date of surgery up to death of disease or the date last seen. Survival estimates would be analysed by the Kaplan-Meier method, and the $\log$ rank test would be used to assess statistical significance. 
Multivariate analysis of prognostic factors would be carried out by the Cox regression model. The SPSS software (SPSS version 21.0, SPSS Inc., Chicago, Illinois, USA) was used for all statistical evaluations.

\section{Results}

Between August 2000 and March 2019, the data relative to 170 patients bearing UCSs were collected; of these, 95 were defined as early-stage disease (stage I-II) based on the histological report at the primary surgery, and thus were included in this study. Forty-four patients were managed by LPT, and 51 patients were managed by MIS. About $75 \%$ of patients have been enrolled in the last 10 years.

Table 1 shows the patients features in the whole series, as well as in the LPT and MIS groups: in the whole series, the median age was 67 years (range 39-88), the median BMI was $28 \mathrm{~kg} / \mathrm{m}^{2}$ (range 19-47), and the vast majority of patients $(N=73,76.8 \%)$ referred comorbidities; age, BMI, and proportion of patients with comorbidities did not differ between the LPT group and the MIS one.

As far as surgical procedures are concerned, the vast majority of patients in the whole series $(N=81,85.3 \%)$ underwent type A hysterectomy; type B1 hysterectomy was less frequently carried out in the MIS group than in the LPT group ( $p$ value $=0.042$ ). In the whole series, assessment of pelvic lymph node status was carried out by lymphadenectomy $(N=47,49.5 \%)$, or sampling $(N=9,9.5 \%)$, while very old and/or unfitted patients, and patients intraoperatively judged to harbour excessive tissue fragility $(N=39)$ were not triaged to pelvic lymph node assessment; there was no difference in the distribution of lymph node procedures between the LPT and the MIS group ( $p$ value 0.83 ). Conversely, the number of pelvic lymph nodes removed was lower in the MIS group (median $=12$, range 2-32) compared to the LPT one (median $=21$, range 2-59) compared ( $p$ value 0.016 ).

Aortic lymph node status assessment was carried out in only 13 patients, due to suspicious lymph nodes at surgery (LPT 8, MIS 5). In the whole series, the median number of aortic lymph nodes removed was six (range 3-27) and did not differ between the two groups. Omentectomy was carried out in only seven patients (7.4\%).

Pathologically assessed stage was: stage IA $(N=43)$, IB $(N=32)$, II $(N=20)$; stage II was more frequent in the MIS group compared to the LPT group, but the $p$ value was not significant ( $p$ value $=0.51$ ). All pelvic and aortic lymph nodes were histologically negative.

The tumor diameter was demonstrated to be larger in the LPT patients compared to the MIS group (median size $7 \mathrm{~cm}$, range 1-20 versus median size $5 \mathrm{~cm}$, range 2-12.5, $p$ value $<0.0001)$. The rate of positive lymphovascular space invasion (LVSI) was shown in $41.5 \%$ of all patients and did not differ between the two groups.

\section{Adjuvant treatment}

As shown in Table 1, in the whole series, 21 patients were triaged to surveillance due to very old age, and/or unsatisfactory performance status $(N=19)$, or refusal $(N=2)$, while 73 patients were administered adjuvant treatment; there was no difference in the distribution of adjuvant treatment between the two groups. Of patients undergoing adjuvant treatment, $15(20.3 \%)$ were administered radiotherapy, 29 (39.2\%) were treated by only chemotherapy, and $30(40.5 \%)$ were treated by chemotherapy followed by radiotherapy. There was no difference in the time interval from completion of surgery and start of adjuvant treatment between the two groups; the median time from surgery to the start of the adjuvant therapy was 6 weeks in both groups (data not shown).

\section{Surgical details and intraoperative and postoperative morbidity}

Surgical details are shown in Table 2: the operative time (OT) was lower in the MIS group versus the LPT group (median $=130 \mathrm{~min}$, range 50-400 versus media $N=180 \mathrm{~min}$, range 60-300) ( $p$ value $=0.021)$; the median estimated blood loss (EBL) was $50 \mathrm{cc}$ (range 10-600) in the MIS group compared to the median of $100 \mathrm{cc}$ (range 100-2500) in the LPT group ( $p$ value $<0.0001$ ) (Table 3 ).

As far as the hospital stay is concerned, the number of days was shorter in the MIS patients (median $=3$, range $2-10)$ versus the LPT group (median $=6.5$, range $2-21)(p$ value $<0.0001)$. Only 1 intra-operative complication was documented: it occurred in the LPT group, and consisted in the lesion of the right obturator vein which was promptly repaired, though resulting in $2500 \mathrm{cc}$ EBL. Overall, there were $(15.0 \%)$ post-operative complications; they were more frequent in the LPT group $(N=7,15.9 \%)$ versus the MIS group $(N=1,2.0 \%)(p$ value $=0.023)$ (Table 2$)$. In particular, in the LPT group there were five surgical complications (grade 2 wound dehiscence, grade lymphocele, grade 3 hemoperitoneum, grade 5 duodenal perforation leading to death, and grade 5 abdominal abscess with death); there were also three medical complications including grade 2 atrial fibrillation, and grade 2 lung failure in the LPT group, and only one grade 2 lower limbs neuropathy in the MIS group.

\section{Clinical outcome}

As for April 2020, follow-up was available in 91 patients due to the loss of data for 4 patients; overall, the median follow-up was 18 months (range 1-212), but patients 
Table 1 Patient characteristics

\begin{tabular}{|c|c|c|c|c|}
\hline & $N$ & $\begin{array}{l}\text { LPT } \\
N .(\%)\end{array}$ & $\begin{array}{l}\text { MIS } \\
N .(\%)\end{array}$ & $p$ value $^{\mathrm{a}}$ \\
\hline All & 95 & 44 & 51 & \\
\hline \multicolumn{5}{|l|}{ Age, years } \\
\hline Median (range) & $67(39-88)$ & $66(50-88)$ & $68(39-88)$ & 0.51 \\
\hline \multicolumn{5}{|l|}{ BMI, $\mathrm{kg} / \mathrm{m}^{2}$} \\
\hline Median (range) & $28(19-47)$ & $28(19-45)$ & $28(22-47)$ & 0.67 \\
\hline \multicolumn{5}{|l|}{ Patients with morbidity } \\
\hline No & 22 & $12(27.3 \%)$ & $10(19.6 \%)$ & 0.28 \\
\hline Yes & 73 & $32(72.7 \%)$ & $41(80.4 \%)$ & \\
\hline Type of hysterectomy ${ }^{\mathrm{b}}$ & & & & 0.042 \\
\hline A & 81 & $34(77.3 \%)$ & $47(92.1 \%)$ & \\
\hline B1 & 14 & $10(22.7 \%)$ & $4(7.8 \%)$ & \\
\hline Pelvic lymph node assessment & & & & $0.83^{\mathrm{c}}$ \\
\hline No & 39 & $19(43.2 \%)$ & $20(39.2 \%)$ & \\
\hline Sampling & 9 & $4(9.1 \%)$ & $5(9.8 \%)$ & \\
\hline Lymphadenectomy & 47 & $21(47.7 \%)$ & $26(51.0 \%)$ & \\
\hline No. of pelvic lymph nodes removed & & & & 0.016 \\
\hline Median (range) & $16(2-59)$ & $21(2-59)$ & $12(2-32)$ & \\
\hline Aortic lymph node assessment & & & & $0.37^{\mathrm{c}}$ \\
\hline No & 82 & $36(81.8 \%)$ & $46(90.2 \%)$ & \\
\hline Sampling & 10 & $7(15.9 \%)$ & $3(5.9 \%)$ & \\
\hline Lymphadenectomy & 3 & $1(2.3 \%)$ & $2(3.9 \%)$ & \\
\hline No. of aortic lymph nodes removed & & & & 0.33 \\
\hline Median (range) & $6(3-27)$ & $8.5(5-27)$ & $6(5-16)$ & \\
\hline Omentectomy & & & & 0.044 \\
\hline No & 88 & $38(86.4 \%)$ & 50() & \\
\hline Yes & 7 & $6(13.6 \%)$ & 1() & \\
\hline Stage & & & & 0.51 \\
\hline IA & 43 & $21(47.7 \%)$ & $22(43.1 \%)$ & \\
\hline IB & 32 & $16(36.4 \%)$ & $16(31.4 \%)$ & \\
\hline II & 20 & $7(15.9 \%)$ & $13(25.5 \%)$ & \\
\hline Tumor diameter, $\mathrm{cm}$ & & & & $<0.0001$ \\
\hline Median (range) & $5.7(1-20)$ & $7(1-20)$ & $5(2-12.5)$ & \\
\hline LVSI & & & & 0.51 \\
\hline No & 56 & $28(63.6 \%)$ & $28(54.9 \%)$ & \\
\hline Yes & 39 & $17(36.4 \%)$ & $22(45.1 \%)$ & \\
\hline Adjuvant treatment & & & & 0.8 \\
\hline No & 21 & $9(20.4 \%)$ & $12(27.3 \%)$ & \\
\hline Yes & 74 & $35(79.5 \%)$ & $39(72.7 \%)$ & \\
\hline Radiotherapy & 15 & 6 & 9 & \\
\hline Chemotherapy & 29 & 17 & 12 & \\
\hline Chemotherapy + radiotherapy & 30 & 12 & 18 & \\
\hline
\end{tabular}

${ }^{a}$ calculated by the $\chi 2$ test or the Fisher's exact test for proportions, and the Wilcoxon rank sum test for continuous data; ${ }^{b}$ according to the Querleu-Morrow classification ${ }^{21}$; ${ }^{c}$ calculated based on no lymph node assessment versus lymph node assessment

LPT: laparotomic surgery, MIS: Minimally invasive surgery; BMI: Body Mass Index

managed by MIS were operated in more recent years compared to the LPT patients (i.e. the percentage of MIS patients operated since 2010 was $93.7 \%$ versus $55.0 \%$ in the LPT group).
Overall, the number of recurrences was 34 (37.4\%). Of these, $16 / 43(37.2 \%)$ were documented in the LPT group, and 18/48 (37.5\%) were in the MIS group. Overall, the most frequent site of recurrence was the central pelvic area 
Table 2 Surgical details, perioperative and post-operative complications

\begin{tabular}{|c|c|c|c|c|}
\hline & All $(N=95)$ & LPT $(N=44)$ & $\operatorname{MIS}(N=52)$ & $p$ value $^{\mathrm{a}}$ \\
\hline \multicolumn{4}{|l|}{ Operative time, $\min$} & \multirow[t]{2}{*}{0.021} \\
\hline Median (range) & $150(50-400)$ & $180(60-300)$ & $130(50-400)$ & \\
\hline \multicolumn{4}{|l|}{ Estimated blood loss, cc } & \multirow[t]{2}{*}{$<0.0001$} \\
\hline Median (range) & $100(10-2500)$ & $200(100-2500)$ & $50(10-600)$ & \\
\hline \multicolumn{4}{|l|}{ Length of hospitalization, days } & \multirow[t]{4}{*}{$<0.0001$} \\
\hline Median (range) & $4(2-21)$ & $6.5(2-21)$ & $3(2-10)$ & \\
\hline Patients with intra-operative complications & 1 & 1 & - & \\
\hline Right obturator vein lesion & 1 & 1 & - & \\
\hline Patients with post-operative complications & 8 & 7 & 1 & $\mathbf{0 . 0 2 3}^{\mathrm{b}}$ \\
\hline \multicolumn{5}{|l|}{ Grade II } \\
\hline Atrial fibrillation & 1 & 1 & - & \\
\hline Lung failure and delirium & 1 & 1 & - & \\
\hline Lower limb neuropathy & 1 & - & 1 & \\
\hline Wound dehiscence & 1 & 1 & - & \\
\hline \multicolumn{5}{|l|}{ Grade III } \\
\hline Pelvic lymphocele requiring drainage & 1 & 1 & - & \\
\hline Hemoperitoneum & 1 & 1 & - & \\
\hline \multicolumn{5}{|l|}{ Grade V } \\
\hline Death (duodenal perforation) & 1 & 1 & - & \\
\hline Death (abdominal abscess) & 1 & 1 & - & \\
\hline
\end{tabular}

${ }^{\mathrm{a}}$ calculated by the Wilcoxon rank sum test for continuous data; ${ }^{\mathrm{b}}$ calculated by the Fisher's exact test
Table 3 Pattern of recurrences

\begin{tabular}{lllll}
\hline & $\begin{array}{l}\text { All patients } \\
N=91\end{array}$ & $\begin{array}{l}\text { LPT } \\
N=43(\%)\end{array}$ & $\begin{array}{l}\text { MIS } \\
N=48(\%)\end{array}$ & $p$ value \\
\hline Recurrence & & & & \\
No & 53() & $27(62.8 \%)$ & $30(62.5 \%)$ & 0.76 \\
Yes & 34() & $16(37.2 \%)$ & $18(37.5 \%)$ & \\
Central pelvic & 11 & 6 & 7 & \\
Lymph node & 8 & 4 & 4 & \\
Distant disease & 9 & 5 & 7 & \\
Unknown & 1 & 1 & - & \\
\hline
\end{tabular}

$(N=13)$ followed by distant sites $(N=12)$, and lymph nodes $(N=8)$ (Table 3).

As shown in Fig. 1a, there was no difference in the disease-free survival (DFS) between the two groups; the 2 -years DFS rate was $57 \%$ in the LPT group versus $62 \%$ in the MIS group ( $p$ value 0.628 ). Death of disease was registered in 31 patients $(34.1 \%)$; the 3 -years OS rate was $59 \%$ in the LPT group versus $63 \%$ in MIS groups $(p$ value $=0.835)$ (Fig. 1b).

Univariate analysis of variables conditioning DFS in the whole series showed that only stage II was associated with

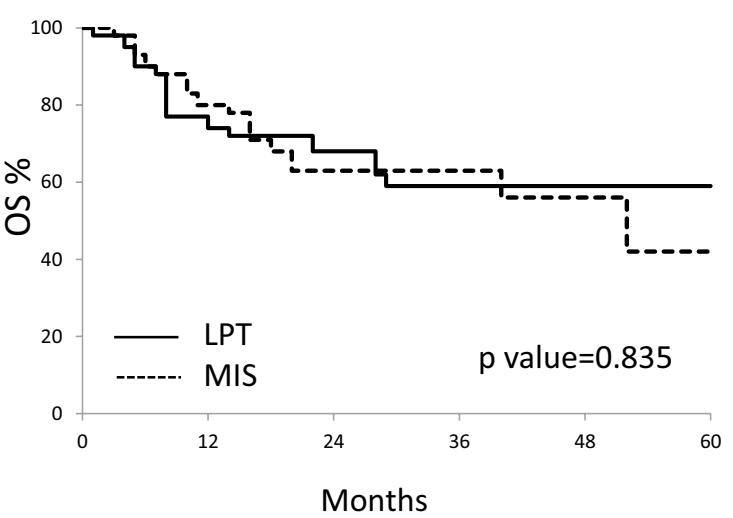

Fig. 1 Kaplan-Meier curves relative to DFS (a), and OS (b) according to the surgical approach 
Table 4 Univariate and multivariate analysis of features conditioning PFS in the whole series $(N=80)$

\begin{tabular}{|c|c|c|c|c|c|c|}
\hline \multirow[t]{2}{*}{ Variable } & \multicolumn{3}{|c|}{ Univariate } & \multicolumn{3}{|c|}{ Multivariate } \\
\hline & $\beta$ & Hazard ratio (CI 95\%) & $p$ value $^{\mathrm{a}}$ & $\beta$ & Hazard ratio (CI 5\%) & $p$ value $^{\mathrm{a}}$ \\
\hline \multicolumn{7}{|l|}{ Age } \\
\hline ( $\leq 67$ years vs. $>67$ years $)$ & 0.349 & $1.418(0.719-2.796)$ & 0.313 & 0.242 & $0.875(0.324-1.902)$ & 0.592 \\
\hline \multicolumn{7}{|l|}{ Stage } \\
\hline (I vs. II) & 1.100 & $3.003(1.495-2.796)$ & 0.002 & 1.551 & $4.715(1.837-12.099)$ & 0.001 \\
\hline \multicolumn{7}{|l|}{ Size of tumor } \\
\hline$(\leq 60 \mathrm{~mm}$ vs. $>60 \mathrm{~mm})$ & 0.567 & $1.762(0.847-3.666)$ & 0.129 & 1.342 & $3.826(1.457-10.047)$ & 0.006 \\
\hline \multicolumn{7}{|l|}{ LVSI } \\
\hline (No vs. yes) & -0.116 & $0.890(0.437-1.812)$ & 0.748 & -0.817 & $0.442(0.186-1.046)$ & 0.079 \\
\hline \multicolumn{7}{|l|}{ Surgical approach } \\
\hline (LPT vs. MIS) & 0.166 & $1.180(0.599-2.326)$ & 0.632 & 0.810 & $2.240(0.850-5.947)$ & 0.102 \\
\hline
\end{tabular}

${ }^{a}$ calculated by Cox regression

LVSI lymphovascular space involvement, MIS minimally invasive surgery, LPT laparotomy

statistically significant worse prognosis ( $p$ value 0.0002 ) (Table 4); in the multivariate analysis, stage II and tumor size were shown to play an independent, unfavorable role (Table 4). Univariate analysis of variables conditioning OS in the whole series showed that only stage II was associated with statistically significant worse prognosis; in the multivariate analysis, stage II and tumor size were associated with unfavorable clinical outcome (data not shown).

\section{Discussion}

UCSs are aggressive tumors, and are included in the "highrisk" group according to the ESMO-ESGO-ESTRO classification (Colombo et al. 2016); however, the clinical outcome of UCSs is even worse than clear cell, and serous EC (Koskas et al. 2016; Fader et al. 2016), thus emphasizing the need to better define the role of MIS focusing on this specific clinical setting. The current NCCN guidelines relative to all "high risk" EC suggest that MIS should be the preferred approach when technically feasible, based on a few retrospective studies (Fader et al. 2012; Koskas et al. 2016; Monterossi et al. 2017; Salehi et al. 2017), and on the subanalysis of the prospective phase III LAP2 trial (Fader et al. 2016). Also, the LACE trial shows as in the endometrial cancer stage I MIS improves the quality of life after surgery, decreases risk of major surgical adverse events without impacting on disease-free survival at 4.5 years and on overall survival (Janda et al. 2010, 2017; Obermair et al. 2012).

However, the studies specifically focused on the role of MIS versus LPT only in UCSs are few, and also include advanced stage of disease (Fader et al. 2012; Koskas et al. 2016; Salehi et al. 2017).

To our knowledge, our study represents the largest, retrospective series comparing the role of MIS versus LPT only in pathologically assessed stage I-II disease UCSs: there was no difference in terms of oncologic outcome between the two approaches, in face of a more favourable peri-operative and post-operative profile in the MIS group.

As far as the surgical procedures are concerned, the rate of type B1 hysterectomy was lower in the MIS patients, a finding which could be ascribed to the smaller tumor diameter in the MIS group, as also suggested in previous studies (Koskas et al. 2016). Assessment of pelvic lymph node status was carried out in about $60 \%$ of overall patients; in this context, we have to acknowledge that $>75 \%$ of our patients had comorbidities, and around one-third were obese, and this could have led to spare patients pelvic lymphadenectomy to limit the operative time, and also reduce surgical morbidity since the high aggressiveness of this histological subtype strongly increases the probability that patients would require adjuvant treatment. Finally, it has been recognized that in the real-world practice, only $35-57 \%$ of patients in some gynecologic oncology centres in USA were triaged to lymphadenectomy, as summarized in the Vorgias review (Vorgias and Fotiou 2010); as a matter of fact, even considering the large series from the SEER database $(N=1885$ patients), and the Netherland Cancer registry $(N=1140$ patients), the "lymphadenectomy issue" still remains controversial (Nemani et al. 2008; Versluis et al. 2018); indeed, the SEER study concluded that lymphadenectomy is associated with improved overall survival with no benefit associated to adjuvant radiotherapy (Nemani et al. 2008), while the Dutch study demonstrates that (1) lymphadenectomy is related to improved survival only if $>10$ lymph nodes are removed, and (2) adjuvant therapy improves survival when lymphadenectomy is omitted, or when lymph nodes are positive (Versluis et al. 2018). Probably, these conflicting findings could be related to the fact that the two studies included also stage III (Nemani 
et al. 2008; Versluis et al. 2018), and even stage IV disease (Versluis et al. 2018). Indeed, in the large series including 5614 stage I UCS patients, lymphadenectomy $\geq 15-20$ lymph nodes removed was associated with better survival (Seagle et al. 2017).

Some recent lines of evidence have been reported relative to the possibility to adopt the sentinel lymph node mapping (SLN) also in UCS patients (Schiavone et al. 2016), as also recommended for low-risk EC (Leitao et al. 2013; Holloway et al. 2017); Indeed, Schiavone et al. (2016) reported similar progression-free survival in UCSC patients (stage I-IV) undergoing SLN and adjuvant therapy compared to systematic lymphadenectomy. The confirmation of these findings in a larger series could lead to guarantee the staging of disease while sparing potential morbidities.

As expected, MIS provided reduced median operative time, and blood loss, and also guaranteed a faster return home, as largely reported (Walker et al. 2009; Fader et al. 2016).

There was only one intraoperative complication in the LPT group; on the other hand, seven (15.9\%) postoperative complications were registered in the LPT group versus only one postoperative complications in the MIS one ( $p$ value $0.023)$ : of these, five were surgical morbidities including three patients with grade 3 morbidities, and two patients experiencing grade 5 morbidities leading to death.

The short hospitalization interval, and the low postoperative morbidity profile may fasten the administration of adjuvant treatment, which was carried out in approximately $75 \%$ of our patients; the distribution of treatments was similar between the two groups, as well as the time interval from surgery to the start of the adjuvant therapy. However, due to the retrospective design of our study, some data about adjuvant therapy were missed, thus representing a bias of the study. In particular, it has been impossible to find accurate radiotherapy data.

As far as the oncologic outcome is concerned, we have to acknowledge that the patients managed by MIS were operated in a more recent time frame, thus leading to shorter follow duration; overall, relapse of disease occurred in was $37.4 \%$, a figure which appears higher compared to the literature; however, the percentage of stage II was higher than expected.

In our series, multivariate analysis led to define stage II of disease, and tumor size as independent, unfavourable prognostic parameters for DFS and OS; this could be ascribed to the fact that this study has focused only on pathologically assessed stage I-II patients, in which the lymph node status and extrauterine disease was not contemplated. Despite the bias potentially associated with the retrospective design and the sample size, we demonstrated that the MIS approach was shown not to be detrimental to the clinical outcome.
Funding Open access funding provided by Università Cattolica del Sacro Cuore within the CRUI-CARE Agreement.

\section{Compliance with ethical standards}

Conflict of interest All the authors report no conflicts of interest. The authors are responsible for the content and writing of the paper.

Open Access This article is licensed under a Creative Commons Attribution 4.0 International License, which permits use, sharing, adaptation, distribution and reproduction in any medium or format, as long as you give appropriate credit to the original author(s) and the source, provide a link to the Creative Commons licence, and indicate if changes were made. The images or other third party material in this article are included in the article's Creative Commons licence, unless indicated otherwise in a credit line to the material. If material is not included in the article's Creative Commons licence and your intended use is not permitted by statutory regulation or exceeds the permitted use, you will need to obtain permission directly from the copyright holder. To view a copy of this licence, visit http://creativecommons.org/licenses/by/4.0/.

\section{References}

Abdulfatah E, Lordello L, Khurram M et al (2019) Predictive histologic factors in carcinosarcomas of the uterus: a multi-institutional study. Int J Gynecol Pathol 38:205-215. https://doi.org/10.1097/ PGP.0000000000000497

Akahira J, Tokunaga H, Toyoshima M et al (2006) Prognoses and prognostic factors of carcinosarcoma, endometrial stromal sarcoma and uterine leiomyosarcoma: a comparison with uterine endometrial adenocarcinoma. Oncology 71:333-340. https://doi. org/10.1159/000107107

Baekelandt MM, Castiglione M (2009) Endometrial carcinoma: ESMO clinical recommendations for diagnosis, treatment and follow-up. Ann Oncol 20:iv29-iv31. https://doi.org/10.1093/annonc/mdp120

Cantrell LA, Blank SV, Duska LR (2015) Uterine carcinosarcoma: a review of the literature. Gynecol Oncol 137:581-588. https://doi. org/10.1016/j.ygyno.2015.03.041

Carlson J, McCluggage WG (2019) Reclassifying endometrial carcinomas with a combined morphological and molecular approach. Curr Opin Oncol 31:411-419. https://doi.org/10.1097/CCO.00000 00000000560

Colombo N, Creutzberg C, Amant F et al (2016) ESMO-ESGOESTRO consensus conference on endometrial cancer: diagnosis, treatment and follow-up. Int J Gynecol Cancer 26:2-30. https:// doi.org/10.1097/IGC.0000000000000609

Creasman W (2009) Revised FIGO staging for carcinoma of the endometrium. Int J Gynecol Obstet 105:109-109. https://doi. org/10.1016/j.ijgo.2009.02.010

Dellinger TH, Wakabayashi MT, Han ES (2017) Comprehensive staging of uterine carcinosarcoma using a multiquadrant robotic platform. J Minim Invasive Gynecol 24:531-532. https://doi. org/10.1016/j.jmig.2016.10.010

Denschlag D, Ulrich UA (2018) Uterine carcinosarcomas-diagnosis and management. Oncol Res Treat 41:675-679. https://doi. org/10.1159/000494335

Fader AN, Java J, Tenney M et al (2016) Impact of histology and surgical approach on survival among women with early-stage, highgrade uterine cancer: an NRG oncology/gynecologic oncology group ancillary analysis. Gynecol Oncol 143:460-465. https:// doi.org/10.1016/j.ygyno.2016.10.016 
Fader AN, Seamon LG, Escobar PF et al (2012) Minimally invasive surgery versus laparotomy in women with high grade endometrial cancer: a multi-site study performed at high volume cancer centers. Gynecol Oncol 126:180-185. https://doi.org/10.1016/j. ygyno.2012.04.028

Gonzalez Bosquet J, Terstriep SA, Cliby WA et al (2010) The impact of multi-modal therapy on survival for uterine carcinosarcomas. Gynecol Oncol 116:419-423. https://doi.org/10.1016/j.ygyno .2009 .10 .053

Holloway RW, Abu-Rustum NR, Backes FJ et al (2017) Sentinel lymph node mapping and staging in endometrial cancer: a society of gynecologic oncology literature review with consensus recommendations. Gynecol Oncol 146:405-415. https://doi. org/10.1016/j.ygyno.2017.05.027

Janda M, Gebski V, Brand A et al (2010) Quality of life after total laparoscopic hysterectomy versus total abdominal hysterectomy for stage I endometrial cancer (LACE): a randomised trial. Lancet Oncol 11:772-780. https://doi.org/10.1016/S1470-2045(10)70145 $-5$

Janda M, Gebski V, Davies LC et al (2017) Effect of total laparoscopic hysterectomy vs total abdominal hysterectomy on disease-free survival among women with stage $\mathrm{i}$ endometrial cancer: a randomized clinical trial. JAMA 317:1224. https://doi.org/10.1001/ jama.2017.2068

Koskas M, Jozwiak M, Fournier M et al (2016) Long-term oncological safety of minimally invasive surgery in high-risk endometrial cancer. Eur J Cancer 65:185-191. https://doi.org/10.1016/j. ejca.2016.07.001

Leitao MM, Khoury-Collado F, Gardner G et al (2013) Impact of incorporating an algorithm that utilizes sentinel lymph node mapping during minimally invasive procedures on the detection of stage IIIC endometrial cancer. Gynecol Oncol 129:38-41. https://doi. org/10.1016/j.ygyno.2013.01.002

Leskela P-M, Rosa-Rosa et al (2019) Molecular basis of tumor heterogeneity in endometrial carcinosarcoma. Cancers 11:964. https:// doi.org/10.3390/cancers11070964

Matsuo K, Ross MS, Machida H et al (2018) Trends of uterine carcinosarcoma in the United States. J Gynecol Oncol. https://doi. org/10.3802/jgo.2018.29.e22

Monterossi G, Ghezzi F, Vizza E et al (2017) Minimally invasive approach in type II endometrial cancer: Is it wise and safe? J Minim Invasive Gynecol 24:438-445. https://doi.org/10.1016/j. jmig.2016.12.022

Nemani D, Mitra N, Guo M, Lin L (2008) Assessing the effects of lymphadenectomy and radiation therapy in patients with uterine carcinosarcoma: a SEER analysis. Gynecol Oncol 111:82-88. https://doi.org/10.1016/j.ygyno.2008.05.016

Obermair A, Janda M, Baker J et al (2012) Improved surgical safety after laparoscopic compared to open surgery for apparent early stage endometrial cancer: results from a randomised controlled trial. Eur J Cancer 48:1147-1153. https://doi.org/10.1016/j. ejca.2012.02.055

Onstad MA, Schmandt RE, Lu KH (2016) Addressing the role of obesity in endometrial cancer risk, prevention, and treatment. J Clin Oncol 34:4225-4230. https://doi.org/10.1200/JCO.2016.69.4638

Plataniotis G, Castiglione M (2010) Endometrial cancer: ESMO clinical practice guidelines for diagnosis, treatment and follow-up. Ann Oncol 21:v41-v45. https://doi.org/10.1093/annonc/mdq245
Pothuri B, Ramondetta L, Eifel P et al (2006) Radiation-associated endometrial cancers are prognostically unfavorable tumors: a clinicopathologic comparison with 527 sporadic endometrial cancers. Gynecol Oncol 103:948-951. https://doi.org/10.1016/j. ygyno.2006.05.039

Querleu D, Morrow CP (2008) Classification of radical hysterectomy. Lancet Oncol 9:297-303. https://doi.org/10.1016/S1470 $-2045(08) 70074-3$

Ross MS, Elishaev E, Berger JL et al (2018) Prognostic significance of omental disease and the role of omental sampling in patients with uterine carcinosarcoma. Int J Gynecol Cancer 28:254-259. https ://doi.org/10.1097/IGC.0000000000001176

Salehi S, Åvall-Lundqvist E, Legerstam B et al (2017) Robot-assisted laparoscopy versus laparotomy for infrarenal paraaortic lymphadenectomy in women with high-risk endometrial cancer: a randomised controlled trial. Eur J Cancer 79:81-89. https://doi. org/10.1016/j.ejca.2017.03.038

Schiavone MB, Zivanovic O, Zhou Q et al (2016) Survival of patients with uterine carcinosarcoma undergoing sentinel lymph node mapping. Ann Surg Oncol 23:196-202. https://doi.org/10.1245/ s10434-015-4612-2

Seagle B-LL, Kanis M, Kocherginsky M et al (2017) Stage I uterine carcinosarcoma: matched cohort analyses for lymphadenectomy, chemotherapy, and brachytherapy. Gynecol Oncol 145:71-77. https://doi.org/10.1016/j.ygyno.2017.01.010

Tan Z, Li A, Chen L et al (2017) Port-site metastasis of uterine carcinosarcoma after laparoscopy. J Korean Med Sci 32:1891. https:// doi.org/10.3346/jkms.2017.32.11.1891

The Cancer Genome Atlas Research Network, Levine DA (2013) Integrated genomic characterization of endometrial carcinoma. Nature 497:67-73. https://doi.org/10.1038/nature12113

U.S. Department of Health and Human Services (2009) Common terminology criteria for adverse events (CTCAE)

Uterine Neoplasms (2020) NCCN Clinical Practice Guidelines in Oncology (NCCN Guidelines $\left.{ }^{\circledR}\right)$ Uterine Neoplasms

Versluis MAC, Pielsticker C, van der Aa MA et al (2018) Lymphadenectomy and adjuvant therapy improve survival with uterine carcinosarcoma: a large retrospective cohort study. Oncology 95:100-108. https://doi.org/10.1159/000488531

Vorgias G, Fotiou S (2010) The role of lymphadenectomy in uterine carcinosarcomas (malignant mixed mullerian tumours): a critical literature review. Arch Gynecol Obstet 282:659-664. https://doi. org/10.1007/s00404-010-1649-0

Walker JL, Piedmonte MR, Spirtos NM et al (2009) Laparoscopy compared with laparotomy for comprehensive surgical staging of uterine cancer: gynecologic oncology group study LAP2. J Clin Oncol 27:5331-5336. https://doi.org/10.1200/JCO.2009.22.3248

Wallwiener C, Hartkopf A, Kommoss S et al (2016) Clinical characteristics, surgical management and adjuvant therapy of patients with uterine carcinosarcoma: a retrospective case series. Geburtshilfe Frauenheilkd 76:188-193. https://doi.org/10.1055/s-0042-100205

Publisher's Note Springer Nature remains neutral with regard to jurisdictional claims in published maps and institutional affiliations. 